\title{
Modeling positive inter-jurisdictional public spending spillovers
}

\author{
Martin Gregor
}

December 12, 2012

\begin{abstract}
This paper builds spatial microfoundations for the functional forms used in the analysis of inter-jurisdictional public spending spillovers. It introduces a symmetric bilateral model that distinguishes between three stages: production of multiple public inputs (intermediary goods), production of multiple public outputs (final goods) including asymmetries and non-additive aggregations, and consumption of the public outputs with asymmetries and preferences for variety. The model is characterized by seven modeling features, and the paper demonstrates how these features are combined in the relevant literature. The paper identifies sufficient conditions for the different combinations of the features to be isomorphic. Additionally, it analyzes which microfoundations for the inter-jurisdictional spillovers lead to asymmetrically structured demands for public spending.
\end{abstract}

Keywords: spillover, externality, complementarity, weak-link, local public goods

JEL Classification: H4, H7 


\section{Introduction}

How should positive public spending spillovers be formally represented? Recently, Levaggi (2010) proposed a simple two-dimensional taxonomy of the inter-jurisdictional public spending spillovers. The first relevant feature is whether public spending covers local public goods that are functionally equivalent or different (homogenous and heterogeneous). This aspect determines whether the domestic and external benefits can be considered additive. The second feature is the structure of weights imposed upon the consumption goods, where the weights are typically interpreted as differentiated access to local public goods across the districts.

How does this spillovers taxonomy treat setups that have appeared so far in the literature? In the dominantly used setup (see Besley and Coate, 2003; Dur and Roelfsema, 2005; Cheikbossian, 2008; Crivelli and Staal, 2012; Feidler and Staal, 2012; Giuranno, 2010; Schnellenbach et al., 2010; Loeper, 2011), each local public good is assumed to produce a decreasing marginal benefit, domestic and external benefits are separable, and benefits of the goods but not the levels of the goods - are weighted by the respective weights. The setup is isomorphic to the treatment used in the environmental literature, where the marginal benefits both from domestic and foreign public spending are assumed constant (hence benefits are linear in the amounts of the local public goods) and the marginal abatement costs of each good are separable and increasing (c.f. Banzhaf and Chupp, 2012).

This classic quasi-linear setup is primarily imposed for the algebraically convenient properties such as zero income effects and separability of benefits. But what are the microfoundations for the separable and decreasing marginal benefits? Levaggi (2010) interprets the setup as a case of preferences for diversity over heterogeneous and differently accessible goods. In this paper, we illustrate that the this explanation contains two interpretations that are mutually inconsistent; the interpretation of the quasi-linear form by means of preferences for diversity is inconsistent with the interpretation of weights by means of accessibility. In this particular example, we will demonstrate that consistent reasoning on the microfoundations of spillovers might reveal contradictions between motivation and assumptions of the models of interjurisdictional spillovers.

Secondly, the two features are not sufficient to represent a rich class of complementary aggregations generating so-called weak-link and weakest-link public goods (see Cornes, 1993; Cornes and Sandler, 1996; Varian, 2004; Cornes and Hartley, 2007). What is missing is the production of intermediary goods that would be aggregated non-additively, where the aggregation may potentially involve spillovers. In the end, the difference between a setup with and without intermediary goods may be technically innocent if a functional form of an extended setup leads to the functional form of a reduced setup. Still, in a reduced setup, the variables capture different things (e.g., they treat intermediary and final goods interchangeably), and this may generate confusion in the interpretation of the building blocks of the setup.

In this paper, we propose a more comprehensive bilateral model of positive interjuris- 
dictional spillovers. The model builds microfoundations around the spatial structures of production and consumption as well as around the properties of the aggregation technology, preferences, and cross-transfers. The model distinguishes between three stages: (i) inputs production with spending vectors instead of scalars, (ii) outputs production with input asymmetries and aggregation, and (iii) consumption with output asymmetries and preferences for variety. We identify seven modeling features that we consider crucial for a comprehensive taxonomy of the spillovers and for each of the features, we provide suitable interpretation. The setup is instrumental in distinguishing between spillovers into production and spillovers into consumption. Through the setup, we can also explicitly determine the source of nonadditive compositions in the presence of spillovers, and properly establish additivity in the aggregation of spillovers with the domestic spending.

The paper proceeds as follows: In Section 2, we formalize the two features proposed by Levaggi (2010) by constructing a baseline model with a single production stage. As a next step, we discuss shortcomings of the baseline model with respect to modeling of interjurisdictional spillovers. Section 3 proposes a comprehensive model, and motivates and interprets key features involved in the model. Section 4 analyzes selected properties of the comprehensive model. We study which particular setups are possible to establish in the comprehensive model and consequently identify isomorphic setups. Next, we identify sufficient and necessary conditions for the existence of asymmetric demands for inputs in symmetric allocations. This is one of the essential problems that can be conveniently analyzed by means of the comprehensive model. That is, we find combinations of the features for which the marginal benefit from the external spending in a symmetric allocation is larger than the marginal benefit from the domestic spending. Section 5 concludes.

\section{Baseline model}

There are two districts, $j=1,2$, each represented by a single representative citizen. Each district pays a local public good at a fixed price $p$, and the amount of the public good in district $j$ is $z_{j}$. In the analysis of pure effects of spillovers, we intentionally disregard any redundant asymmetry. Hence, we keep utility functions $U$, input prices $p$ and initial incomes $m$ identical.

$$
U_{j}=U\left(F\left(z_{j}, z_{-j}\right) ; m-p z_{j}\right)
$$

There is an aggregation function $F(\cdot)$ of the local public goods. In all what follows, we consider only positive spillovers, i.e. $\frac{\partial F_{j}}{\partial z_{j}} \geq 0$ and $\frac{\partial F_{j}}{\partial z_{-j}} \geq 0$. In the baseline model, two modeling features are involved in the construction of the function:

- Asymmetry: A parameter $\kappa \in[0,1 / 2]$ reflects access to consumable benefits associated with domestic versus foreign goods. There are two extremes. For $\kappa=0$, there is no access to the foreign good. In other words, there is full asymmetry in the treatment 
of the domestic and foreign goods. For $\kappa=1 / 2$, both local public goods are treated identically, and there is full symmetry in the goods.

- Aggregation: Levaggi (2010) attributes the shape of the aggregation to goods heterogeneity. If the local public goods have a constant marginal rate of transformation, they behave are pure substitutes. This is interpreted as homogeneity of the goods. In contrast, if the local public goods have a variable marginal rate of transformation, there is the case of heterogeneous goods with the citizens having preferences for variety.

Levaggi (2010) proposes $F_{j}=F\left(z_{j}, z_{-j}\right)=(1-\kappa) f\left(z_{j}\right)+\kappa f\left(z_{-j}\right)$. If $f(\cdot)$ is linear, we have homogenous goods, and for $f(\cdot)$ non-linear (increasing and concave), we have heterogeneous goods. Out of the two characteristics, Levaggi (2010) constructs three different setups: (i) No spatial effect $(\kappa=0)$ defines a setting with two local public goods without spillovers. (ii) Symmetric and additive effect ( $\kappa=1 / 2$, homogeneity) defines a global public good. (iii) Local public goods with spillovers $(\kappa \in(0,1 / 2)$ for homogeneity; $\kappa \in(0,1 / 2]$ for heterogeneity) capture the existence of two possibly different local public goods with spillovers.

Where are the limits of this basic classification? We provide three motivating points:

- Incompleteness. There is a class of global public goods called weak-link and weakest-link public goods. Production of these goods features complementary aggregation (Cornes, 1993; Cornes and Sandler, 1996). In the baseline model, it would be represented by $\kappa=1 / 2$ and a non-linear $f(\cdot)$. Complementarity would have to be attributed to goods heterogeneity. But, in the case of a single global public good, there is no heterogeneity in the final goods; what is heterogeneous are intermediary goods that are produced in the districts. Clearly, one must (i) separate the production process into the production of intermediary and final goods, and also (ii) consider the option of contributing to multiple intermediary goods. The first feature affects mainly the functional form. The second feature affects also the strategy sets of the players, and introduces the importance of the structure of each district's spending, not only of its overall level.

- Motivation. Does the presented baseline model actually describe the situations that motivate its construction? Consider the stylized quasi-linear form $F\left(z_{j}, z_{-j}\right)=(1-$ $\kappa) f\left(z_{j}\right)+\kappa f\left(z_{-j}\right)$. As argued above, the quasi-linear functional specification is attributed to the preferences for diversity and the parameters $(1-\kappa, \kappa)$ are seen as fixed access (share) parameters. ${ }^{1}$ Yet there are at least two possibilities how to build microfoundations for the setup, depending on where we draw the line between the production and consumption: (i) If the weights $(1-\kappa, \kappa)$ indeed represent access, they must multiply the consumable amounts of the local public goods. Here, the consumable amounts

\footnotetext{
${ }^{1} \mathrm{An}$ example is a theater used partly by visitors from neighboring districts; we can assume that the shares of visitors are constant irrespective of the number of plays.
} 
are $f\left(z_{j}\right)$ and $f\left(z_{-j}\right)$ and they enter the utility additively, hence the representative citizen has no preference for diversity over the local public goods. In other words, the decreasing marginal benefit of public spending $z_{j}$ or $z_{-j}$ should be interpreted as the decreasing marginal productivity in the local production function, not as a property of the preferences, because the function $f(\cdot)$ does not characterize preferences over the goods but production of a consumable good. (ii) Alternatively, if the preferences are over heterogeneous consumable goods, then all access parameters should be part of the argument of $f(\cdot)$ function. But there are no such parameters in the argument. The weights $(1-\kappa, \kappa)$ should not be interpreted as access parameters but as parameters capturing district-specificity of tastes. More concretely, the weights represent homebias towards consumption of the domestic local public good. To summarize, the setting can be interpreted either as preferences for a homogenous good with different accessibility to the good across the districts, or as preferences for heterogenous goods, with a relatively larger utility attached to the domestic good.

- Lacking microfoundations for the key properties. The aggregation function $F(\cdot)$ is often constructed arbitrarily since it is difficult to fit it from the data. To constrain its shape, it typically must satisfy axioms that are intuitively supposed to hold. Consider an axiomatic property that the marginal benefit of domestic spending is less than the marginal benefit of foreign spending, for any combination of the payments. It is intuitively believed that by restricting $\kappa \leq 1 / 2$, this immediately becomes true. But once we apply the preference for variety and access shares consistently (i.e., we multiply the arguments of $f(\cdot)$ by the parameters), and use any well-behaving function, then this property is not guaranteed. Consider $G\left(z_{j}, z_{-j}\right)=\log \left((1-\kappa) z_{j}\right)+\log \left(\kappa z_{-j}\right)$. Here, the foreign spending brings a larger marginal benefit to the district than the domestic spending whenever there is even a tiny excess of the domestic spending relative to the foreign spending, specifically if $z_{j}>z_{-j}$.

To sum up, to argue for a particular functional form requires us to distinguish between the consumption stage and the multiple stages of production. Secondly, we must identify at what stage the asymmetric access and complementarity should be introduced. The interaction between the asymmetries and complementarities must also be carefully addressed. An axiomatic approach that imposes the properties by assumption constitutes a plausible alternative to microfoundations, but if a model seeks to describe a particular case that has well defined microfoundations, then it is reasonable to incorporate these microfoundations explicitly. The drawback of the axiomatic approach is that it is often imposed purely for the sake of convenience, i.e., to secure interior solutions and minimize non-linearities.

Finally, to avoid possible confusions: For local public spending, two distinct sets of properties are relevant. One set of properties materializes within the district, and determines the nature of local public spending as traditionally analyzed (e.g., rivalry and excludability with 
respect to citizens within the districts). These are properties that we do not model or discuss here, since for us, each district is represented by a single agent. We focus only upon properties across the districts. Given territorial and legal reasons, these may be different from the properties within the districts. For example, for some benefits, only domestic citizens are eligible for consumption. Thus, while consumption may be fully non-excludable within the district, it may be fully excludable across the districts. ${ }^{2}$

\section{Comprehensive model}

\subsection{Assumptions}

We augment the baseline model by separating the production process into two stages and proposing direct preferences over the final goods, not only indirect preferences over the spending levels. There are two district-specific inputs, $i=1,2$. Payments by district $j=1,2$ into the inputs are $z_{j}=\left(z_{j, 1}, z_{j, 2}\right)$. Payments made by the districts to the provision of a single input are of course additive, and the amount of the $i$-input is $x_{i}=f\left(z_{1, i}+z_{2, i}\right)$, where $f(\cdot)$ is increasing and concave, i.e., the marginal cost is increasing and convex. For illustration, the public inputs in a district can be the amounts of police personnel in the district, or the number of items in municipality libraries. There are two district-specific outputs aggregated from the inputs, $X_{1}$ and $X_{2}$. Each district consumes the outputs in the form of a composite public good $G_{j}\left(X_{j}, X_{-j}\right)$, and the underlying utility function is $U_{j}=U\left(G_{j}, m-z_{j, 1}-z_{j, 2}\right)$.

The following seven features will be analyzed:

- Inputs production: The production function $f(z)$ is either linearly or non-linearly increasing. We do not consider the possibility that the production of one input is intertwined with the production of another input. Thus, in the argument of the function, there are only payments made to the single input. In the analysis, it is convenient to consider a class of $f(z)=z^{e}$, where $e \in(0,1]$. Whether $e=1$ or $e<1$ holds distinguishes between constant and increasing marginal cost of the inputs (linearity vs. non-linearity). In this particular class, for any $e^{\prime} \neq e$, we have $f^{\prime}(z)=f(z)$ if and only if $z=0$ or $z=1$.

- Inputs specialization: A district is either allowed or not allowed to make a cross-district payment. If such a payment is not feasible, then $z_{j, i}=0$ constraint binds whenever $i \neq j$. An indicator variable $I \in\{0,1\}$ is set to $I=0$ if and only if $z_{1,2}=z_{2,1}=0$ binds.

- Outputs production, access to inputs: In the production of a district-specific output, there might be a privileged access to the domestic district-specific input. Let $(1-k, k)$ be the division by which the district-specific inputs are accessible by the districts, where

\footnotetext{
${ }^{2}$ Of course, there are natural links between these two sets of properties, but how exactly are these two sets linked is left for other research.
} 
$k \in[0,1 / 2]$. In other words, we obtain the levels of the effective inputs $\left((1-k) x_{j}, k x_{-j}\right)$ for the $j$-district that produces $j$-output.

- Outputs production, aggregation: The effective inputs are aggregated into outputs by an aggregation technology that treats effective outputs anonymously. In the literature, a typical class of aggregations are CES-functions, where the output produced in a district $j$ is $X_{j}^{r}=\left[(1-k) x_{j}\right]^{r}+\left[k x_{-j}\right]^{r}$. For $r=1$, we have perfect substitution. For $r \in[0,1)$, we have imperfect substitution. For $r<0$, we have complementarity that approaches perfect complementarity with $r \rightarrow-\infty$.

- Consumption, access to outputs: When consuming the outputs, a district may have privileged access to the output produced in the district. Let $(1-\kappa, \kappa)$ be the division by which the district-specific outputs are consumed by the districts, where $\kappa \in[0,1 / 2]$. In other words, we obtain effective outputs at levels $\left((1-\kappa) X_{j}, \kappa X_{-j}\right)$ for the $j$-district.

- Consumption, aggregation: For the representative citizen, the differences between the outputs are not only in the accessibility, but also in their nature. When the citizen treats the goods as heterogeneous, he or she aggregates the effective outputs in a nonadditive way. For simplicity, we again use the class of CES-functions, with elasticity now characterized by the parameter $\rho$. Again, the function treats effective outputs anonymously. The composite public consumption of $j$-district writes

$$
G_{j}=\left\{\left[(1-\kappa) X_{j}\right]^{\rho}+\left[\kappa X_{-j}\right]^{\rho}\right\}^{\frac{1}{\rho}} .
$$

- Substitution of private and public consumption: For the analysis, it is useful to restrict the behavior of the marginal rate of substitution between the private and public consumption in the $U(\cdot)$ function. The most frequent form is quasilinear: both types of benefits are separable, but one of the goods enters in an increasing and concave function and the other good enters linearly. We will consider only utilities strictly increasing in both arguments.

The final functional form is as follows. For $j$-district,

$$
U_{j}=U\left(\left\{\left[(1-\kappa) X_{j}\right]^{\rho}+\left[\kappa X_{-j}\right]^{\rho}\right\}^{\frac{1}{\rho}}, m-z_{j, 1}-z_{j, 2}\right),
$$

where

$$
X_{j}^{r}=\left[(1-k) x_{j}\right]^{r}+\left[k x_{-j}\right]^{r} .
$$

and

$$
x_{j}=f\left(z_{j, j}+z_{-j, j}\right) .
$$

Any comprehensive setup that employs CES-aggregations is characterized as a seven-tuple $\{f, I, k, r, \kappa, \rho, U\}$. 


\subsection{Motivation and interpretations}

Two functions with spatial characteristics are involved: a spatial output production function and a spatial consumption function. The logic and motivation behind spatial properties of each of the two functions can be different. This section analyzes motivation for the spatial features and discusses their properties.

- Inputs production. We focus only on linearity and non-linearity of the cost function, i.e. on whether the marginal cost of an input is increasing or not. Most frequently, the marginal cost is set constant because the government purchases are not assumed to be that large to affect the marginal productivity and the unit price on the entire market. This typical partial-equilibrium assumption is realistic for municipalities, but it may be inappropriate for the interactions on the global levels, e.g. when large states contract large purchases with a few suppliers. Non-linearity forces the equilibrium towards more symmetry, since largely asymmetric amounts of inputs in the presence of a fixed marginal rate of transformation of the inputs generate the opportunities for the cost arbitrage. The magnitude of the effect crucially depends on the possibility to make cross-district payments.

- Domain of payments. Often, the specialization restriction is imposed because crosspayments are irrelevant in the equilibrium. When a local input brings a larger marginal benefit irrespective of the profile of payments, specialization into domestic inputs is an equilibrium property, and the model is robust to the possibility to make a cross-payment. If this property does not hold, the restriction may matter substantially. Vicary (1990) started a literature that investigates in-kind transfers to the weakest-link public goods and shows the high strategic importance of the cross-transfers (see Sandler and Vicary, 2001; Vicary and Sandler, 2002; Lei et al. 2007; Gregor, 2011). In a companion paper (Gregor and Stastna, 2012), we demonstrate that with the specialization to domestic inputs and under complementary spillovers, increasing the amount of spillovers paradoxically increases the equilibrium public spending in non-cooperative decentralization. Specialization restriction may stem from the combination of territorial aspects of the production and administrative and legal barriers to in-kind transfers. For example, if the production of inputs involves armed forces, then each district will maintain its territorial control over the inputs and provision of the inputs is hardly contractible. In such a case, the only feasible non-cooperative transfer is a cash transfer, not an in-kind transfer. But a cash transfer is typically less effective in motivating production of the extra inputs because of the income effect that produces leakage to private good consumption. For quasi-linear utility where private good spending is linear (a very frequent specification), this income effect implies full leakage to private spending, and cash transfers are completely ineffective in raising extra inputs in the other district. Thus, the 
combination of infeasible in-kind transfers and quasi-linear utility forces the districts to specialize on payments to the domestic inputs.

- Access to the inputs. The parameter $k$ is interpreted as the access to the foreign input (input spillover or spill-in), and partial access reflects imperfect appropriability of the inputs and mobility costs of the producers of the outputs. An alternative interpretation could be that domestic inputs are better utilized than foreign inputs. This is possible to say for an output production function that is symmetric (anonymous) in the effective inputs, not in the nominal inputs. In this interpretation, $k$ can be viewed as a technology parameter. An example is tacit local knowledge that improves productivity or utilization of the domestic inputs.

- Aggregation of the effective inputs. It is frequently the case that the good provided either by the market or the public sector is not the final good, but an intermediary good, and the consumer needs another complementary intermediary good to produce the final good by himself or herself. For example, consider the density and quality of a railway network in two districts; the citizen's decision to use railway transportation in both districts (e.g., for commuting) will be more dependent on the marginal improvements in density and quality in a worse district than in a better district. In addition to such technical complementarities, the literature on the weak-link and weakest-link public goods motivates complementary aggregation by protection against adversaries who more likely tend to attack the weaker parts than the stronger parts of a network. In our case, the parts are represented by the effective amounts of the inputs.

- Access to the outputs. The level of the parameter $\kappa$ that is interpreted as access is related to the degree of excludability, to the possibility of discrimination between the consumers from two districts, and to mobility costs.

- Aggregation of the effective outputs. If the outputs represent heterogeneous consumption goods, the citizen is normally having preferences where the marginal rate of substitution is increasing in the ratio of the amounts of the two goods. This is the case of the utility in variety.

\subsection{Multiple interpretations of the quasilinear form}

Two setups with different microfoundations may lead to an exactly identical functional form. Consider the quasilinear case discussed in the introduction, namely $F\left(z_{j}, z_{-j}\right)=(1-\kappa) f\left(z_{j}\right)+$ $\kappa f\left(z_{-j}\right)$. The fixed linear weights imply that the final-stage aggregation must be linear, hence any non-linear (non-additive) aggregation must be introduced in earlier stages. We have only two earlier stages, therefore non-additivity is present either in the production of inputs or in the production of outputs. This difference also determines at which stage the spillovers are present: 
- Non-additive inputs, spillovers in the production stage (input spillovers): Suppose that each input is produced non-linearly, $x_{i}=f\left(z_{i}\right)$. In the production of outputs, there is partly asymmetric access $(1-\kappa, \kappa)$ and additive aggregation of the effective inputs, $X_{j}=(1-\kappa) x_{j}+\kappa x_{-j}$. In consumption, there is fully asymmetric access, $G_{j}=X_{j}$, hence aggregation does not take place.

- Non-additive outputs, spillovers in the consumption stage (output spillovers): Suppose that each input is produced linearly, $x_{i}=z_{i}$. In the production of outputs, there is fully asymmetric access, and the production function is non-linear, $X_{j}=f\left(x_{j}\right)$. In consumption, there is partly asymmetric access $(1-\kappa, \kappa)$ and the aggregation is additive, $G_{j}=(1-\kappa) X_{j}+\kappa X_{-j}$.

In both interpretations, separability renders the marginal products independent. Strictly speaking, the heterogeneity issue is not related to the aggregation into an intermediary input or to preference for a more balanced composition of the goods, but only to the marginal costs of the additive components $f\left(z_{j}\right)$ and $f\left(z_{-j}\right)$. The reason for obtaining relatively balanced amounts in the equilibrium is in the decreasing returns to scale which motivate substitution from unbalanced production levels to more balanced production levels. Another similarity between the setups is redundancy. Yet, the redundant stages are different in the two setups. For input spillovers, the inputs-production stage must be inevitably introduced, but consumption stage becomes redundant. For output spillovers, the inputs-production stage is redundant, but consumption stage is necessary.

Note that in our setting, we have limited the interpretation of the asymmetric parameters either to access or to the presence of a third, district-specific factor of production. There can be other microfoundations for the asymmetry, but these would violate our interest in functions that are anonymous in the effective inputs and effective outputs. ${ }^{3}$

\footnotetext{
${ }^{3}$ How could these microfoundations be introduced? For spillovers in the production stage, the production function may be district-specific. Namely, a domestic input is considered to be directly more productive than a foreign input. Think of noisy information gathered by police investigations. A piece of local knowledge may be more informative if priors over the domestic fundamentals are sharper than priors over the foreign fundamentals. For spillovers in the consumption stage, think of the following: Once we allow for preference heterogeneity, we may interpret the weights as preference parameters (e.g., home bias). In this article, we limit asymmetries only to access parameters that have only spatial or technological nature. One reason is that spatial asymmetries in preferences must be result of some exogenous process that requires to be modeled (such as sorting), and a proper account for such heterogeneity has to incorporate the locational choices which is not the topic of our analysis.
} 


\section{Properties}

\subsection{Isomorphic setups}

The example in the previous subsection has illustrated possible equivalence of two otherwise differently motivated setups. More generally, how do we identify that two setups are isomorphic? We will examine a strong form of isomorphism that requires that for any given profile of payments, the players' consumption bundles in the two setups do not differ from each other.

Definition 1 (Isomorphism) We consider two setups $A=\left\{f_{A}, I_{A}, k_{A}, r_{A}, \kappa_{A}, \rho_{A}\right\}$ and $B=\left\{f_{B}, I_{B}, k_{B}, r_{B}, \kappa_{B}, \rho_{B}\right\}$ isomorphic if for any admissible $\left(z_{1}, z_{2}\right)$ and $j=1,2, G_{A, j}\left(z_{1}, z_{2}\right)=$ $G_{B, j}\left(z_{1}, z_{2}\right)$.

Notice that the indicator variable is irrelevant in this definition of the isomorphisms; the indicator variable only changes admissibility of certain contribution profiles. In a weak definition of isomorphisms where we could essentially study invariance of the equilibrium to parametric changes, indicator variable would certainly matter. This problem will be discussed in the subsequent section. Also, for our definition, the shape of the utility function is not incorporated in the analysis, hence implicitly $U_{A}(\cdot)=U_{B}(\cdot)$.

Two propositions on strong isomorphisms are constructed. In Proposition 1, we will see that in the presence of additivity in both stages, isomorphisms exist within this class of fully additive setups. Namely, any linear combination of access parameters in the output and consumption stages can be replaced by a different linear combination of the two access parameters. Proposition 2 identifies a special case of 'switching isomorphism' in another class of cases, namely if there is a single stage without spillovers. In such a case, two setups are isomorphic if and only if they switch the production and consumption stages.

Proposition 1 (Isomorphisms for full additivity) If output production and consumption stages with access parameters $\left(k_{A}, \kappa_{A}\right)$ are additive in a setup $A$, i.e., $r_{A}=\rho_{A}=$ 1 , then any setup $B$ with different access parameters $\left(k_{B}, \kappa_{B}\right) \neq\left(k_{A}, \kappa_{A}\right)$ and other features unchanged, $\left(f_{A}, r_{A}, \rho_{A}, U_{A}\right)=\left(f_{B}, r_{B}, \rho_{B}, U_{B}\right)$, is isomorphic if a linear constraint $\kappa_{B}+k_{B}-2 \kappa_{B} k_{B}=\kappa_{A}+k_{A}-2 \kappa_{A} k_{A}$ holds, and admissibility constraints are met, i.e., $k_{B}, \kappa_{B} \in[0,1 / 2]$.

In Proposition 2, we consider the case of having at least a single stage fully asymmetric. We disregard differences in the input production, $f_{A}(\cdot)=f_{B}(\cdot)$. In the fully asymmetric stage, the aggregation parameter is irrelevant, hence among plausible isomorphisms, we consider setups that differ in more aspects than in the irrelevant aggregation parameter (generic difference). The proposition shows that such a unique (generically different) isomorphic setup exists. It is characterized such that the properties of the production stage replace properties of the consumption stage and vice versa. 
Proposition 2 (Isomorphisms for full asymmetry) Consider a setup A which involves at least one fully asymmetric stage, i.e., $k_{A}=0$ or $\kappa_{A}=0$. A generically different setup $B$ that keeps other features unchanged, $\left(f_{A}, U_{A}\right)=\left(f_{B}, U_{B}\right)$, is isomorphic if and only if $k_{i}=\kappa_{-i}$ and $k_{i} r_{i}=\kappa_{i} \rho_{-i}$, where $i=A, B$.

\subsection{Classification of the main alternatives}

In the literature, we recognize two primary alternatives: the classic quasilinear setup (Besley and Coate, 2003; BC03) and the setup with complementary spillovers (Gregor and Stastna, 2012; GS12). Table 1 compares the two setups and shows isomophisms with other closely related combinations. The classification of setups is based on the features involved in our comprehensive model. In each stage (output production and consumption), we distinguish between three options: (i) fully asymmetric access, regardless of the aggregation, (ii) partially asymmetric access with additivity and (iii) partially asymmetric access with non-additivity (complementarity).

Table 1: Taxonomy of the most frequent setups

\begin{tabular}{|c|c|c|c|c|}
\hline Outputs production & Consumption & Model & Inputs productions & Cross-payments \\
\hline Full asymmetry & Full asymmetry & Private goods & Linearity/non-linearity & Always irrelevant \\
\hline Full asymmetry & Additivity & Besley, Coate (2003) & Non-linearity & Irrelevant in eq. \\
\hline Full asymmetry & Additivity & Levaggi (2010) & Linearity & Irrelevant in eq. \\
\hline Full asymmetry & Complementarity & Isomorphic to GS12 & Linearity & Key feature \\
\hline Additivity & Full asymmetry & Isomorphic to BC03 & Non-linearity & Irrelevant in eq. \\
\hline Additivity & Additivity & Linearly transformed BC03 & Non-linearity & Irrelevant in eq. \\
\hline Complementarity & Full asymmetry & Gregor, Stastna (2012) & Linearity & Key feature \\
\hline
\end{tabular}

Normally, the classification of goods covered by public spending is based on non-rivalry and non-excludability and not on the features covered in our comprehensive model. From that perspective, there are four extreme cases (a global public good, commons, non-rival but excludable/appropriable goods, and pure local goods) and a continuum of intermediate cases. The question is whether these are the properties that may characterize the setups better than those covered in our taxonomy.

First, consider excludability in consumption. Clearly, when the marginal rate of transformation of inputs is considered, an equally relevant issue to excludability of the consumption of the final goods is the structure access to the intermediary goods. Second, for rivalry, notice that we can transform rivalry into non-rivalry and vice versa if we examine only the relative use of the goods. In other words, if we have a unit of either input or output, then a rival case assumes spatial use of the inputs in rival $1-\kappa$ and $\kappa$ shares. The non-rival case assumes spatial use of the inputs in non-rival shares 1 and $\sigma$. If only the relative use matters, then the two cases are identical if $\sigma=\frac{\kappa}{1-\kappa}$, or if $\kappa=\frac{\sigma}{1+\sigma}$. To summarize, in the analysis of the inter-jurisdictional spillovers, the two classic features of rivalry and excludability are not of higher-order importance than the features modeled in our setting. 


\subsection{Treatment of the inputs in the equilibrium}

For the two players, the model is symmetric, hence a symmetric equilibrium exists. In a symmetric allocation, the amounts of inputs are identical, $x_{1}=x_{2}$, and also outputs are identical $X_{1}=X_{2}$. For such symmetric allocations, does district $j$ treat differently the domestic input $j$ and foreign input $-j$ ? Namely, does district $j$ demand relative more of input $j$ than input $-j$ in the equilibrium? Does the fact that a spillover effect is less than the domestic effect (i.e., $k<1 / 2$ or $\kappa<1 / 2$ ) imply a bias to finance domestic inputs?

In this section, we will investigate whether the inputs are demanded symmetrically or asymmetrically, and if asymmetrically, whether district $j$ in a symmetric allocation demands relatively more of input $j$ or not. This issue is relevant for the issue of the existence of domestic specialization and for the question if the possibility of cross-payments matters in the equilibrium. Gregor and Stastna (2012) show that in the presence of input and output asymmetries and non-additive aggregation of the inputs, the existence of cross-payments matters for the shape of the asymmetry in the equilibrium demands. But which combinations of non-additivities and asymmetries and in which stages are relevant and which are not?

To find symmetry or asymmetry in the relative demands, we introduce a $j$-player's marginal rate of transformation (MRT) of inputs $\left(x_{j}, x_{-j}\right)$ in the production of the composite good $G_{j}: M R T_{j,-j}^{j}(\cdot):=\frac{\partial G_{j}}{\partial x_{j}} / \frac{\partial G_{j}}{\partial x_{-j}}$. We can proceed in either of two equivalent ways. We may evaluate the MRT in the symmetric allocations $x=x_{1}=x_{2}$. If $M R T_{j,-j}^{j}(x, x)=1$, then the demands are symmetric. If $M R T_{j,-j}^{j}(x, x)>1$, then the domestic input is relatively more productive. If $M R T_{j,-j}^{j}(x, x)<1$, then the foreign input is relatively more productive. Equivalently, we may identify for which $\left(x_{j}, x_{-j}\right)$ is $M R T_{j,-j}^{j}\left(x_{j}, x_{-j}\right)=1$. If $x_{j}>x_{-j}$, then we must have $M R T_{j,-j}^{j}(x, x)>1$ (the domestic input is relatively more productive). If $x_{j}<x_{-j}$, then we must have $M R T_{j,-j}^{j}(x, x)<1$ (the foreign input is relatively more productive). Below, we will apply the latter approach.

As a first step, we will see that asymmetry in inputs is a necessary (but not sufficient) condition for any demand asymmetry. Take symmetry in inputs, $k=1 / 2$. Then, outputs are identical for any $\left(x_{1}, x_{2}\right): X:=X_{1}=X_{2}$. Consumption is also identical for both players, $G_{1}^{\rho}=(1-\kappa)^{\rho} X^{\rho}+\kappa^{\rho} X^{\rho}=G_{2}^{\rho}$. Thus, both players treat the inputs in the same way, and there is no difference between the players in the relative demands for inputs, irrespective of the composition of inputs $\left(x_{1}, x_{2}\right)$. Since each aggregation is from identical outputs, each player's consumption aggregation is irrelevant for the MRT of inputs, and it is sufficient to study the relative productivities of inputs in the production of the common output. Thanks to inputs symmetry, however, it is equal to one if $x_{1}=x_{2}$, irrespective of the aggregation parameter $r$. Thus, both players demand the inputs identically in the symmetric allocations.

We are left with $k<1 / 2$. How to combine the aggregations with asymmetry or asymmetries? For simplification, we do not work with two complementarities in two different stages 
at the same time. ${ }^{4}$ Then, there are six options listed in Table 2 :

Table 2: Cases of input asymmetry and at most single complementarity

\begin{tabular}{lccc}
\hline Case & Outputs production $(r, k)$ & Consumption $(\rho, \kappa)$ & Preferred input \\
\hline C1 & Additive asymmetry & Additive symmetry & Both \\
C2 & Additive asymmetry & Additive asymmetry & Domestic \\
C3 & Additive asymmetry & Complementary symmetry & Both \\
C4 & Additive asymmetry & Complementary asymmetry & Foreign \\
C5 & Complementary asymmetry & Additive symmetry & Both \\
C6 & Complementary asymmetry & Additive asymmetry & Foreign \\
\hline
\end{tabular}

- Additive stages (C1, C2). By Proposition 1, in the case of additivities $(r=\rho=1)$, generically different setups are characterized only single-dimensionally by the unique value of linearly combined access weights. In other words, all setups with identical $k+\kappa-2 k \kappa$ are isomorphic. It is easy to find the relative demands. Consider any $(k, \kappa)$ such that $k<1 / 2$. For any $\left(x_{1}, x_{2}\right)$,

$$
M R T_{j,-j}^{j}\left(x_{j}, x_{-j}\right)=\frac{Z_{1}(\kappa, k)}{Z_{2}(\kappa, k)}=\frac{(1-\kappa)(1-k)+\kappa k}{\kappa(1-k)+(1-\kappa) k} .
$$

Note that $Z_{1}(\kappa, k)>Z_{2}(\kappa, k)$ is equivalent to $(1-\kappa)(1-2 k)>\kappa(1-2 k)$, and since $k<1 / 2$, it is equivalent to $1-\kappa>\kappa$ which holds always for $\kappa<1 / 2$. If $\kappa=1 / 2$ (C1), then $G_{1}=G_{2}$ and the inputs are thanks to symmetry of $X_{j}$ and $X_{-j}$ treated identically, $M R T_{j,-j}^{j}(x, x)=1$. If $\kappa<1 / 2(\mathrm{C} 2)$, then each district prefers and specializes on the domestic input relative to the foreign input, $M R T_{j,-j}^{j}(x, x)>1$.

- Complementary consumption (C3, C4). There is input asymmetry $k<1 / 2$ in the additive production of the outputs, $X_{j}=(1-k) x_{j}+k x_{-j}$. Start with the more simple case $\mathrm{C} 3$ where the outputs be treated identically in the complementary aggregation, $\kappa=1 / 2$. (The outputs can be seen as two heterogeneous global public goods.) Then, $G_{j}^{\rho}=\left[X_{j} / 2\right]^{\rho}+\left[X_{-j} / 2\right]^{\rho}=G_{-j}^{\rho}$ and $G_{j}=G_{-j}$ irrespective of the aggregation. The MRT is equal one if

$$
X_{j}^{\rho-1}(1-k)+X_{-j}^{\rho-1} k=X_{j}^{\rho-1} k+X_{-j}^{\rho-1}(1-k)
$$

which for $k<1 / 2$ requires $X_{j}=X_{-j}$ and this requires for $k<1 / 2 x_{j}=x_{-j}$. Thus, irrespective of the asymmetry in the downstream production stage, the districts' demands

\footnotetext{
${ }^{4}$ This extension can be easily built and does not affect the main results.
} 
for inputs are in the symmetric allocations aligned, and the existence of asymmetry does not generate any wedge in the districts' input demands. In fact, C3 only generalizes C1 by allowing for arbitrary $\rho$; in both cases, symmetry in consumption implies identity in the composite consumption good.

For $\kappa<1 / 2$ (case C4), the situation is different. The MRT is equal one if

$$
(1-\kappa)^{\rho} X_{j}^{\rho-1}(1-k)+\kappa^{\rho} X_{-j}^{\rho-1} k=\kappa^{\rho} X_{j}^{\rho-1} k+(1-\kappa)^{\rho} X_{-j}^{\rho-1}(1-k),
$$

which requires for $k<1 / 2$

$$
\frac{X_{j}}{X_{-j}}=\frac{(1-k) x_{j}+k x_{-j}}{k x_{j}+(1-k) x_{-j}}=\left(\frac{1-\kappa}{\kappa}\right)^{\frac{\rho}{1-\rho}}<1 .
$$

When speaking of complementarity, we apply sufficiently large complementarity, $\rho<0$. The inequality $X_{j}<X_{-j}$ in (9) is satisfied if and only if $x_{j}<x_{-j}$. Thus, a district relatively prefers the foreign input when the inputs are asymmetric and additive and the outputs are asymmetric and complementary.

- Complementary production and additive consumption (C5, C6). For the marginal rate of transformation of the inputs to be equal one, we must have

$$
(1-2 \kappa)(1-k)^{r} x_{j}^{r-1}=(1-2 \kappa) k^{r} x_{-j}^{r-1} .
$$

If the outputs are weighed identically in the additive consumption stage, $\kappa=1 / 2$, (C5), then the equality in (10) holds for any $\left(x_{1}, x_{2}\right)$. Intuition is clear: The outputs are different, but exactly like in $\mathrm{C} 1$ and $\mathrm{C} 3$, the composite consumption goods are identical. Any restructuring of inputs results in a marginal increase in one output that exactly offsets a marginal decrease in the other output. The exact offset is given by the fact that the two outputs are weighted identically.

If access to the domestic output is better than access to the foreign output $(\kappa<1 / 2$, case C6), then the equality in (10) holds if

$$
\frac{x_{j}}{x_{-j}}=\left(\frac{1-k}{k}\right)^{\frac{r}{1-r}} .
$$

For sufficiently large complementarity, $r<0$, this gives $x_{j}<x_{-j}$. In this configuration, if a district is allowed to pay for any input, then each district prefers to replace the excessive domestic inputs for the missing foreign inputs.

Proposition 3 summarizes the results, which are also put into the last column of Table 2 .

Proposition 3 (Demand for foreign input) In a comprehensive model with at most single non-additivity, a district in a symmetric equilibrium strictly prefers the foreign input if and only if the access in both production and consumption stages is asymmetric and either the production or consumption stage is complementary. 


\section{Conclusions}

Inter-jurisdictional public spillovers are present in at least two forms, in the production of the territorially specific goods and in the consumption of these territorially specific goods. The production phase involves positive spillovers when public inputs from various districts can be accessed. The consumption phase involves positive spillovers when public outputs from various locations can be accessed. The two phases may use different aggregation functions and different access parameters.

In this paper, we introduce a symmetric bilateral model that distinguishes between three stages: production of the multiple public inputs (intermediary goods), production of the multiple public outputs (final goods) accounting for asymmetries and aggregation, and consumption of the public outputs with asymmetries and preferences for variety. The main benefit of the comprehensive model is that the real world examples of spillovers can be better classified. The model also illustrates that assumptions of particular models should be imposed with large care to avoid misinterpretations and to secure that the modeling setup accurately describes the case of interest.

The setup is intentionally limited to the analysis of the simplest issues in the spatial production and consumption. Namely, we offer a purely symmetric and only bilateral setting. More complex spillovers such as networks or higher-order spillovers (Bloch and Zenginobuz, 2007) cannot be analyzed. Heterogeneity is suppressed as well. We disregard otherwise relevant asymmetries such as the initial levels of the public goods, asymmetric costs, and asymmetric productivities. Possible differences in indirect utility functions over public spending are attributed only to objective spatial characteristics, not to tastes, hence the model abstracts from the interaction of spatial and technological characteristics with the locational choices of the heterogeneous individuals. While these extensions are relevant, our view is that the structure of production and consumption has to be understood in the first step before other issues are investigated. Only this understanding allows us to disentangle effects that stem from the spatial dependence and aggregation from effects which are related to exogenous district heterogeneities.

\section{Acknowledgements}

I would like to thank an anonymous referee for valuable comments. Financial support by the Czech Science Foundation (P402/12/G09766) is gratefully acknowledged. 


\section{A Proofs}

Proof of Proposition 1. The premises in the proposition disregard any potential change in the inputs production, hence $f_{A}(z)=f_{B}(z)$. In district 1 , by additivity:

$$
\begin{aligned}
& G_{A, 1}=\left(1-\kappa_{A}\right) X_{1}+\kappa_{A} X_{2}=\left(1-\kappa_{A}\right)\left[\left(1-k_{A}\right) x_{1}+k_{A} x_{2}\right]+\kappa_{A}\left[k_{A} x_{1}+\left(1-k_{A}\right) x_{2}\right]= \\
& =\left(1+2 \kappa_{A} k_{A}-\kappa_{A}-k_{A}\right) x_{1}+\left(\kappa_{A}+k_{A}-2 \kappa_{A} k_{A}\right) x_{2}=Z_{1}\left(\kappa_{A}, k_{A}\right) x_{1}+Z_{2}\left(\kappa_{A}, k_{A}\right) x_{2} .
\end{aligned}
$$

We have expressed the amount of the composite public output as the function of two linear combinations, $Z_{1}(\kappa, k)$ and $Z_{2}(\kappa, k)$. For an additive setup $B$ with access parameters $\left(k_{B}, \kappa_{B}\right)$ to be isomorphic to setup $A$, it must necessarily be that $Z_{1}\left(\kappa_{A}, k_{A}\right)=Z_{1}\left(\kappa_{B}, k_{B}\right)$ and $Z_{2}\left(\kappa_{A}, k_{A}\right)=Z_{2}\left(\kappa_{B}, k_{B}\right)$. From these equalities, we obtain

$$
\kappa_{B}=\frac{1-k_{B}-Z_{1}\left(\kappa_{A}, k_{A}\right)}{1-2 k_{B}}
$$

and

$$
\kappa_{B}=\frac{Z_{2}\left(\kappa_{A}, k_{A}\right)-k_{B}}{1-2 k_{B}} .
$$

By imposing equality, we obtain $1-Z_{1}\left(\kappa_{A}, k_{A}\right)=Z_{2}\left(\kappa_{A}, k_{A}\right)$ that holds irrespective of the values of $k_{B}$. Thus, if the value $k_{B}$ is arbitrarily (only respecting the standard admissibility bounds) and $\kappa_{B}$ is set in line with the conditions above, then the two setups are isomorphic.

Proof of Proposition 2. Sufficiency. Consider the two different setups $A, B$. From the first condition, let w.l.o.g. $k_{A}=\kappa_{B}=0$ and $k_{B}=\kappa_{A} \geq 0$. The second condition then does not restrict $\left(r_{A}, \rho_{B}\right)$ because both are multiplied by zero (both are irrelevant), and only requires $r_{B}=\rho_{A}$. We have:

$$
\begin{aligned}
G_{A, j}\left(x_{1}, x_{2}\right)=\left[\kappa_{A} X_{j}^{\rho_{A}}+\left(1-\kappa_{A}\right) X_{-j}^{\rho_{A}}\right]^{1 / \rho_{A}}=\left[\kappa_{A} x_{j}^{\rho_{A}}+\left(1-\kappa_{A}\right) x_{-j}^{\rho_{A}}\right]^{1 / \rho_{A}} \\
G_{B, j}\left(x_{1}, x_{2}\right)=X_{j}=\left[k_{B} x_{j}^{r_{B}}+\left(1-k_{B}\right) x_{-j}^{r_{B}}\right]^{1 / r_{B}}=\left[k_{B} x_{j}^{r_{B}}+\left(1-k_{B}\right) x_{-j}^{r_{B}}\right]^{1 / r_{B}}
\end{aligned}
$$

Since $\kappa_{A}=k_{B}$ (by the first condition) and $r_{B}=\rho_{A}$ (by the first and second conditions), we have $G_{A, j}\left(x_{1}, x_{2}\right)=G_{B, j}\left(x_{1}, x_{2}\right)$.

Necessity. First, $A$ setup may involve both stages fully asymmetric. Then, $G_{j}=X_{j}=x_{j}$. An isomorphic setup $B$ must also involve full asymmetry in both stages, but then it is not generically different to $A$. Second, setup $A$ involves a single fully asymmetric stage. W.l.o.g., we proceed with the case of $k_{A}=0$ and $\kappa_{A}>0$. Then, $G_{A, j}\left(x_{1}, x_{2}\right)$ is a CES-aggregation of $\left(x_{1}, x_{2}\right)$ with weights $\left(\kappa_{A}, 1-\kappa_{A}\right)$ and elasticity parameter $\rho_{A}$. For isomorphism, $G_{B, j}\left(x_{1}, x_{2}\right)$ must also be a CES-aggregation of $\left(x_{1}, x_{2}\right)$ with the identical weights and the identical parameter. But, if two not-fully asymmetric CES-aggregations are compositely aggregated, then one cannot receive a CES-aggregation of the linearly weighted inputs $\left(x_{1}, x_{2}\right)$. (This can be 
seen from investigating the marginal products in the full form.) Only by setting one stage fully asymmetric, one can receive a CES-aggregation. Thus, we must have either $k_{B}=0$ or $\kappa_{B}=0$.

Now, we will establish the claim that if the stage that is asymmetric is identical in setups $A$ and $B$ (i.e., if $k_{B}=k_{A}=0$ ), then we would require also $\kappa_{B}=\kappa_{A}$ and $r_{B}=r_{A}$ for isomorphism, and the setups would not be generically different. To see that $\kappa_{B}=\kappa_{A}$ and $r_{B}=$ $r_{A}$ is indeed required for isomorphism, think of two CES-aggregations, $V_{j}\left(x_{j}, x_{-j}\right)^{r}=[(1-$ $\left.k) x_{j}\right]^{r}+\left[k x_{-j}\right]^{r}$ and $W_{j}\left(x_{j}, x_{-j}\right)^{s}=\left[(1-m) x_{j}\right]^{s}+\left[m x_{-j}\right]^{s}$. When do we have $V_{j}\left(x_{j}, x_{-j}\right)=$ $W_{j}\left(x_{j}, x_{-j}\right)$ for any $x_{j}, x_{-j}$ ? By separability and investigating marginal effects in $x_{j}$ and $x_{-j}$, the equivalence requires $\left[(1-k) x_{j}\right]^{r}=\left[(1-m) x_{j}\right]^{s}$ and $\left[k x_{-j}\right]^{r}=\left[m x_{-j}\right]^{s}$. Notice that we can disregard intercepts because $V_{j}(0,0)=0=W_{j}(0,0)$ for any admissible quadruplet $(r, s, m, k)$. Take the first term: $x_{j}^{r-s}=(1-m)^{s}(1-k)^{-r}$. Hence, RHS is constant in $x_{j}$. For LHS to be constant, we must have $r=s$. This implies that LHS is equal one. To have RHS also equal one, we must have $1-m=1-k$, hence $k=m$. Thus, the aggregations are equivalent if and only if $r=s$ and $k=m$.

Since setting $\kappa_{B}=\kappa_{A}$ and $r_{B}=r_{A}$ would imply two setups that are not generically different (the only difference could be in the functionally irrelevant parameters $\rho_{A}, \rho_{B}$ ), the asymmetry must be in different stages. Namely, $\kappa_{B}=k_{A}=0$. We then obtain $G_{B, j}\left(x_{1}, x_{2}\right)$ as expressed in (13). Again, by considering separability and investigating marginal effects in $x_{j}$ and $x_{-j}$, we must for securing $G_{A, j}\left(x_{1}, x_{2}\right)=G_{B, j}\left(x_{1}, x_{2}\right)$ impose both $\kappa_{A}=k_{B}$ and $r_{B}=\rho_{A}$. This is summarized in the first and the second conditions.

Proof of Proposition 3. Sufficiency is from C4 and C6, and necessity is from observing that in the remaining cases $\mathrm{C} 1-\mathrm{C} 3$ and $\mathrm{C} 5$, the domestic input is either preferred strictly or both inputs are found equivalently productive.

\section{References}

[1] Banzhaf, H.S., Chupp, B.A. (2012) Fiscal federalism and interjurisdictional externalities: New results and an application to US Air pollution, Journal of Public Economics 96, 449-464.

[2] Besley, T., Coate, S. (2003) Centralized versus Decentralized Provision of Local Public Goods: A Political Economy Analysis, Journal of Public Economics, 87, 2611-37.

[3] Bloch, F., Zenginobuz, U. (2007) The effect of spillovers on the provision of local public goods, Review of Economic Design, 11, 199-216.

[4] Cheikbossian, G. (2008) Rent-seeking, spillovers and the benefits of decentralization, Journal of Urban Economics, 63, 217-228. 
[5] Cornes, R. (1993) Dyke maintenance and other stories: some neglected types of public good. Quarterly Journal of Economics, 107, 259-271.

[6] Cornes, R., Hartley, R. (2007) Weak links, good shots and other public good games: building on BBV. Journal of Public Economics 91 (9), 1684-1707.

[7] Cornes, R., Sandler, T. (1996) The Theory of Externalities, Public Goods and Club Goods. Cambridge: Cambridge University Press.

[8] Crivelli, E., Staal, K. (2012) Size, spillovers and soft budget constraints. International Tax and Public Finance, forthcoming, DOI 10.1007/s10797-012-9230-3.

[9] Dur, R., Roelfsema, H. (2005) Why does centralisation fail to internalise policy externalities? Public Choice, 122, 395-416.

[10] Feidler, J., Staal, K. (2012) Centralized and decentralized provision of public goods. Economics of Governance, 13 (1), 73-93.

[11] Giuranno, M.G. (2010) Pooling sovereignty under the subsidiary principle, European Journal of Political Economy, 26, 125-136.

[12] Gregor, M. (2011) Tradeoffs of foreign assistance for the weakest-link global public goods. International Tax and Public Finance, 18 (2), 233-251.

[13] Gregor, M., Stastna, L. (2012) The decentralization tradeoff for complementary spillovers, Review of Economic Design, 16(1), 41-69.

[14] Lei, V., Tucker, S., Vesely, F. (2007). Foreign aid and weakest-link international public goods: An experimental study. European Economic Review, 51 (3), 599-623.

[15] Levaggi, R. (2010) From local to global public goods: How should externalities be represented? Economic Modelling, 27, 1040-1042.

[16] Loeper, A. (2011) Coordination in heterogeneous federal systems, Journal of Public Economics, 95, 900-912.

[17] Sandler, T., Vicary, S. (2001) Weakest-link public goods: giving in-kind or transferring money in a sequential game. Economics Letters, 74, 71-75.

[18] Schnellenbach, J., Feld, L.P., Schaltegger, C. (2010) The impact of referendums on the centralisation of public goods provision: a political economy approach, Econonomics of Governance, 11, 3-26.

[19] Varian, H.R. (2004) System reliability and free riding. In: L. Jean Camp and Stephen Lewis (eds.), Economics of Information Security, Advances in Information Security, Vol. 12, Springer, 1-15.12 
[20] Vicary, S. (1990) Transfers and the weakest-link: An extension of Hirshleifer's analysis. Journal of Public Economics, 43, 375-394.

[21] Vicary, S., Sandler, T. (2002) Weakest-link public goods: giving in-kind or transferring money. European Economic Review, 41, 1506-1520. 